\title{
Margins in DFSP Reconsidered: Primum Non Nocere
}

\author{
Winan J. van Houdt \\ Department of Surgical Oncology, The Netherlands Cancer Institute, Amsterdam, The Netherlands
}

Editorial discussing "Conservative Re-excision Is a Safe and Simple Alternative to Radical Resection in Revision Surgery for Dermatofibrosarcoma Protuberans"

Dermatofibrosarcoma protuberans (DFSP) is a dermal sarcoma and is, therefore, a tumor that lies at the interface between a sarcoma and a skin tumor. Due to the rarity of this disease, it is often not recognized by general healthcare providers but resected without a pretreatment biopsy, assuming the lesion to be a benign skin lesion. After these so-called whoops operations, further reexcision in order to obtain clear margins is considered standard of care. These reexcisions are usually performed with wide margins, up to 2-4 cm, based on local protocols and guidelines, leading to more morbid operations frequently requiring some form of reconstruction. In the current study from the Royal Marsden in London, Snow et al. describe that conservative reexcision-meaning with only small margins —of these postexcision scars is often sufficient for disease control, when there is no clinical or radiological evidence of macroscopic residual disease. ${ }^{1}$ The paper does not aim to make new recommendations for the minimal width of the reexcision margin, but the take-home message is rather to tailor surgical strategies by weighing disease biology, anatomical location, and individual patient characteristics to balance between optimal oncological control and preventing morbid operations. The story of margins in sarcomas in general, and DFSP specifically, is not a onesize-fits-all protocol prescribing a 1-, 2-, or 3-cm margin, but a story of personalizing surgical and nonsurgical treatment strategies considering all relevant factors.

(C) Society of Surgical Oncology 2019

First Received: 13 October 2019;

Published Online: 4 November 2019

W. J. van Houdt

e-mail: w.v.houdt@nki.nl
There have been many debates regarding treatment of DFSP, especially discussing the technique of operation and the width of the resection margins. ${ }^{2}$ To better understand this discussion, it is important to be aware of the setting in which these debates take place. First of all, since DFSP is a rare disease, many physicians and surgeons understandably have a lack of knowledge regarding this disease, leading to under- or overtreatment of this disease. Also, the surgical approach to this tumor varies widely depending on the specialty of the surgeon the patient is referred to. Since it is a dermal tumor, some patients go to dermatologists for further treatment, while other patients are referred to general surgeons or even plastic surgeons or ear, nose, and throat (ENT) specialists. Another group is referred to specialized sarcoma centers, sometimes for the primary treatment but more often after inadequate treatment elsewhere. There have been extensive discussions, and sometimes even animosity, between the different specialties regarding the best surgical techniques and referral patterns. Each specialty deals with the disease in its own manner and has preferences regarding how to treat DFSP patients. Many surgeons consider all DFSPs to be true sarcomas and resect these tumors aggressively, aiming to achieve wide pathological resection margins, making it more likely to need skin grafts or flap reconstructions for closure. Sometimes resections are even combined with radiotherapy when the pathological margins are not wide enough according to local standards and protocols. At the other end of the spectrum, dermatologists are keen to perform Mohs surgery, and many dermatological studies have been published stating that Mohs surgery leads to fewer recurrences and is the treatment of choice. Also, independently of the surgical technique, DFSP patients are often followed intensely with chest imaging and frequent clinical visits, as if they were all true malignant sarcomas.

Although the discussions regarding the surgical techniques and margins will probably never grow quiet, I would like to emphasize that real progress for the patients 
will not be made by debates regarding surgical techniques or specific rules for margin width, but rather by increased understanding of the biological behavior of the disease as well as awareness of the limitations of every treatment approach. It is becoming increasingly clear that DFSPs without any signs of fibrosarcomatous (FS) changes are most likely not capable of metastasizing. ${ }^{3}$ In contrast, DFSPs with FS can mimic the behavior of truly high-grade sarcomas. Therefore, the latter, relatively small cohort of patients with FS should be treated and followed as any other high-grade sarcoma patient. However, when no FS is diagnosed by a specialist sarcoma pathologist, DFSPs can be considered as locally aggressive, precancerous lesions, but probably not as a true sarcoma with metastatic potential. ${ }^{4}$ This important distinction highlights that all DFSPs should be diagnosed or reviewed by an expert pathologist with ample experience with DFSP, and every pathology report should mention whether there is FS present or not.

Since positive resection markers are a predictive factor for recurrence, the goal of surgery should be primarily to obtain pathological clear margins for any surgical technique. However, clear margins can only be confirmed after reliable assessment of the margins. Pathological sampling and margin assessment should therefore be done with care by meticulous evaluation of the margins by experienced pathologists. Lack of expertise and lack of standardization leave room for error, and this might affect local recurrence rates more than the specific surgical technique applied or the surgical margin achieved.

When smaller DFSP tumors without FS are resected with clear margins, they are very unlikely to develop local recurrences. ${ }^{4}$ The exact definition of clear margins varies widely, as well as opinions regarding the ideal width of the surgical and pathological margins. It is clear that the pathological margin is often smaller than the surgical margin, probably because DFSPs can grow with tentacles spreading beyond the macroscopic tumor. Therefore, 2-3$\mathrm{cm}$ margins are often considered to be sufficient surgical margins. However, the wider the surgical margins, the more likely it is that reconstructive surgery will be needed, varying between split skin grafts and reconstructive flaps, potentially leading to morbidity and unfavorable esthetic results. Anatomical location is an important factor to take into account, since wide excisions with primary closure are easier to carry out at the proximal lower extremity or the abdominal skin, whereas in other regions, such as the head and neck, wide excisions can be truly morbid. For this reason, Mohs surgery in the head and neck regions might be a better option than wide local excision. Recently, we published a paper in Cancer showing that, in small tumors without FS resected with clear pathological margins of 1 $\mathrm{mm}$ or more, none of the patients developed a recurrence, suggesting that pathological margins of $1 \mathrm{~mm}$ or more seem wide enough to prevent local recurrences.

Snow et al. teach us that, after an unplanned excision with macroscopic complete resection of the tumor, a conservative reexcision is often enough to obtain clear margins leading to excellent local control. In all these patients, potential morbid surgery by taking wide margins of $3 \mathrm{~cm}$ or more was prevented. These encouraging data might convince other surgeons and dermatologists to be more conservative in their reexcisions and do no further harmprimum non nocere. Of note, it could even be argued that, when a recurrence is easy to treat at the anatomical location where the tumor was excised, one could even wait with revision surgery for a potential recurrence before performing a (re)excision. Recently, the French sarcoma group reported no effects on overall survival for patients with superficial sarcomas that were observed and not reexcised after unplanned excisions. ${ }^{5}$

Nevertheless, macroscopic residual tumor after unplanned resections and tumors with fibrosarcomatous changes may require a different approach by taking wider margins. But, even in these tumors, all relevant factors need to be taken into account before performing major morbid resections.

In summary, the following strategy could be considered: After unplanned excisions, reexcision of scars when no macroscopic disease is present can be treated with conservative reexcision. However, when there is clinical or radiological macroscopic disease present, wider margins compared with primary DFSP might be needed.

Snow et al. describe that, in a small number of patients, further reexcisions after first conservative reexcision were necessary and, in a few cases, even more than one. Obviously, this can be a burden for patients which should be discussed with patients beforehand, since for some patients this might not be a suitable strategy. However, since for most patients one conservative reexcision was sufficient, morbid surgery for reexcisions can be prevented and should therefore be encouraged.

Some open questions remain, such as the role of imatinib in larger tumors and whether neoadjuvant imatinib can lead to smaller resections. Also, follow-up in non-FS patients might be omitted in patients with good prognostic factors due to the very low risk of recurrence, ${ }^{4}$ although this is not confirmed in prognostic studies.

However, the main take-home message is that optimal outcome for DFSP patients depends on the ability of the surgeon to carefully navigate between Scylla and Charybdis, meaning her or his ability to navigate between preventing harmful large operations and obtaining optimal oncological control, taking tumor biology, anatomical location, and patient characteristics into account. The 
challenge for surgeons when treating DFSP patients might therefore not be the surgical resection per se, but rather to take a step back and reconsider all options.

\section{REFERENCES}

1. Snow H, Davies E, Strauss DC, Smith M, Hayes AJ. Conservative re-excision is a safe and simple alternative to radical resection in revision surgery for dermatofibrosarcoma protuberans. Ann Surg Oncol. 2019 (in press).

2. Foroozan M, Sei JF, Amini M, Beauchet A, Saiag P. Efficacy of Mohs micrographic surgery for the treatment of dermatofibrosarcoma protuberans: systematic review. Arch Dermatol. 2012;148(9):1055-1063.

3. Fiore M, Miceli R, Mussi C, Lo Vullo S, Mariani L, Lozza L, et al. Dermatofibrosarcoma protuberans treated at a single institution: a surgical disease with a high cure rate. J Clin Oncol. 2005;23(30):7669-7675.

4. Huis In 't Veld EA, van Coevorden F, Grunhagen DJ, Smith MJ, van Akkooi ACJ, Wouters $\mathrm{M}$, et al. Outcome after surgical treatment of dermatofibrosarcoma protuberans: Is clinical followup always indicated? Cancer. 2019;125(5):735-741.

5. Decanter G, Stoeckle E, Honore C, Meeus P, Mattei JC, DubrayLongeras $\mathrm{P}$, et al. Watch and wait approach for re-excision after unplanned yet macroscopically complete excision of extremity and superficial truncal soft tissue sarcoma is safe and does not affect metastatic risk or amputation rate. Ann Surg Oncol. 2019;26(11):3526-3534.

Publisher's Note Springer Nature remains neutral with regard to jurisdictional claims in published maps and institutional affiliations. 